International Journal of Forensic Science \& Pathology (IJFP)

ISSN 2332-287X

\title{
Langerhans Cell - The Destination of Migration
}

Short Communication

Sugiura $K^{*}$, Sugiura M

Department of Environmental Dermatology and Allergology, Daiichi Clinic, 2F Nittochi Nagoya building, 2-1-1 Sakae, Nakaku, Nagoya, Aichi, Japan.

\section{Abstract}

Langerhans cells (LCs) capture irritant substances in the skin, and then LCs migrate to the lymph nodes for presenting antigens. For investigating this, we used mites antigen labeled with PKH26 dye in atopic model NC mice for patch testing. The readings of skin reactions were done at $0.5,1,3,6,9,24,48,72$ and 168 hours after the patch testing. At 24-, 48-, 72- and 168-hour, some LCs expressed PKH26 dye were observed in the sinus, T-cell area and marginal area of the regional lymph nodes. The B-cell areas of these lymph nodes were shown as dark and round-shaped at the same time points. By electron microscopy, we found rod-shaped BGs (atypical granules) in the Golgi area of these lymph nodes. We recognized again and showed that LCs with antigen migrate to the lymph nodes.

Keywords: Langerhans Cell; Migration; Lymph Nodes.

\section{*Corresponding Author:}

Keiji Sugiura MD, PhD,

Department of Environmental Dermatology and Allergology, Daiichi Clinic, 2F Nittochi Nagoya building, 2-1-1 Sakae, Nakaku, Nagoya, Aichi, 460-0008, Japan.

Tel/Fax: +81-52-204-0835

E-mail: ksugiura@daiichiclinic.jp

Received: July 16, 2015

Accepted: July 30, 2015

Published: August 05, 2015

Citation: Sugiura K, Sugiura M (2015) Langerhans Cell - The Destination of Migration. Int J Forensic Sci Pathol. 3(8), 152-155. doi: http:// dx.doi.org/10.19070/2332-287X-1500037

Copyright: Sugiura $\mathbf{K}^{\circ}$ 2015. This is an open-access article distributed under the terms of the Creative Commons Attribution License, which permits unrestricted use, distribution and reproduction in any medium, provided the original author and source are credited.

\section{Introduction}

Dendritic cells (DCs) exist in the lymph nodes, tonsils, blood vessels, lymph vessels, liver, skin, and other tissues for playing essential roles in immune systems. The kinds of DCs are Dermal Dendritic cells (DDCs), Langerhans cells (LCs) in the skin, Interdigitating Dendritic cells (IDCs) in the paracortical area, and Follicular Dendritic cells (FDCs) in the germinal center area.

LCs are antigen-presenting cells (APCs) in the skin that compose about $3-5 \%$ of the epidermis [1]. LCs are originated from bone marrow, and LCs have some granules possessing abilities of migration $[2,3]$. LCs seems to play a redundant function in contact hypersensitivity [4]. In chronic contact hypersensitivity, presenting applied hapten to T cells require LCs [5]. When irritant substances go into the skin, LCs capture the irritant substances and migrate to the draining lymph nodes as they mature, and then LCs present the irritant peptide to T cells [6-9]. Now, CXCR4 [10], CCR7 [10] MDR-1 [11], IL-1 $\beta[12,13]$ and TNF- $\alpha[13]$ are known as related factors for migration and maturation of LCs.

For studying the destination of migration, we used atopic model mice (NC mice) and a Dp (mite) antigen. NC mice (Figure 1) are nishiki-nezumi with a cinnamon color; they develop dermatitis similar to atopic dermatitis (AD) from 5-8 weeks of age under conventional circumstances $[14,15]$. NC mice are considered an animal model of $\mathrm{AD}$ based on histological findings of the skin, laboratory findings and skin barrier function [14-16]. Mites are one causative factor of AD. Previous reports described that an IgE-mediated Type I allergy to one kind of mites contributed to the development of AD-like skin eruptions on NC mice [17, 18]. Our studying showed that not only IgE-mediated immediate allergy but also delayed-type hypersensitivity to the mites would also be partly responsible for the skin eruptions in NC mice [17, 18]. We conducted patch testing, which is a frequently used for identifying delayed-type hypersensitivity (Type IV) allergic skin reactions.

Figure 1.

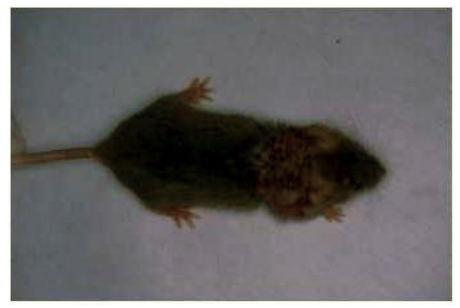

\section{Materials and Methods}

For patch testing, we used Dp (mites) antigen (a mixture of Dermatophagoides pteronyssinus and Dermatophagoides pteronyssing) labeled with PKH26 dye (ZYNAXIS CELL SCIENCE INC., Malvern, PA, USA) in six NC mice. The readings of skin reactions were 
done at $0.5,1,3,6,9,24,48,72$ and 168 hours after the patch testing according to Draize's criteria [19]. All six of the NC mice showed positive reactions at the 24- to 72 - hour readings, demonstrating that the NC mice obtained delayed-type hypersensitivity to the mites. We obtained some skin specimens from the patch-testing sites and regional lymph nodes (inguinal and axial) at each reading time point. We fixed these skin and lymph nodes in acetone at $4^{\circ} \mathrm{C}$, and processed in acetone methyl benzoate xylene (AMeX). We cut them at $2-4 \mu \mathrm{m}$, and stained by hematoxylin-eosin. We used PKC (protein kinase Type C) -II [20] as a marker for LCs by immunohistochemical demonstration. Immunohistochemical study performed using the avidin-biotinperoxidase complex (ABC: Vectastain ABC kit, Vector Lab. Inc., Burlingame, CA, USA) method and indirect methods. For electron microscopically studying, small skin specimens were fixed in a mixture of $2.5 \%$ glutaraldehyde and $2 \%$ paraformaldehyde in $0.1 \mathrm{~N}$-cacodylate buffer, and then post-fixed in $2 \%$ osmium tetroxide buffered with phosphate buffer. We cut these specimens with a diamond knife on LKB ultratome, and stained with uranyl acetate and lead citrate. We examined with a Jeolco 100 CX electron microscope.

Then we homogenate the small skin specimens by the glass and ultrawave-homoginator with the ISOGEN-LS (Wako Pure Chemical Industries Ltd., Osaka, Japan) and stored for 5 minutes. And then we centrifuged subjects at $12 \mathrm{~K} \times \mathrm{g}, 10$ minutes, $4^{\circ} \mathrm{C}$. The aqueous phase was submitted to estimate the protein of GM-CSF (R \& D Systems Inc., MN, USA) by western blotting.

Dates of LCs in the epidermis were presented as means \pm SD. Patch testing site with $\mathrm{Dp}$ and white petrolatum at 48 and 72 hours were compared statistically by student's t-test. $\mathrm{P}<0.05$ were considered significant.

\section{Results and Discussion}

Skin changes were observed as eosinophils and lymphocytes infiltrating into the epidermis and dermis at the 3-hour reading sites. Histological examinations at 48- and 72-hour readings revealed the perivascular infiltration of many lymphocytes and some mast cells in the upper dermis, intracellular edema of the epidermis, lymphocyte infiltration to the epidermis, and liquefaction-degeneration of the basal layer.
We also investigated LCs in an immunohistochemical study using protein kinase C-II (PKC-II) staining as a marker of LCs [20]. Weinlich reported that an insignificant decrease of 10-20\% LCs at one hour after application of contact allergens was evident [21]. The results of our immunohistochemical study showed that LCs had migrated to the dermis at the 1-hour readings, and that type IV allergic skin reaction started from at least 0.5-1 hour. Very few LCs (significantly decreased) were noted in the epidermis at the 48- (by $13.7 \% ; p<0.05$ ) and 72 -hour readings sites (by $17.6 \%$; $p$ $<0.05)$ compared to the negative control at the same time points (Table 1). The number of LCs increased from 72 hours, and many LCs in the skin were replenished from bone marrow at the 168hour sites (Table 1).

At 24-, 48-, 72- and 168-hour, some LCs expressed PKH26 dye were observed in the sinus, T-cell area (Figure $2 \mathrm{a}$ ) and marginal area (Figure $2 \mathrm{~b}$ ) of the regional lymph nodes. The B-cell areas of these lymph nodes were shown as dark and round-shaped (Figure 2a) at the same time points. Kobayashi [22] reported that LCs with Birbeck granules (BGs) migrated into the paracortical area of draining lymph nodes following the injection of sterile saline into the skin. Kripke [23] reported that Ia- and FITC-positive cells from the skin, at least some of which were LCs, left the skin after epicutaneous sensitization with FITC and participated in the initiation of a contact hypersensitivity reaction within the regional lymph nodes. In chronic contact hypersensitivity, presenting applied hapten to T cells require LCs [5]. Thus we demonstrated that LCs with irritant peptide migrate to the lymph nodes, marginal, sinus and $\mathrm{T}$ cell area, by using delayed hypersensitivity skin reactions.

The features of LCs are racket-shaped Birbeck granules (BGs) and rod-shaped atypical granules. These are cytoplasmic granules in the Golgi area; their functions are unknown. Shamoto reported that atypical granules were present in immature LCs [24]; Shamoto noted that atypical granules are going to turn into BGs with the maturation of LCs [24]. By electron microscopy, we found that PKC-II positive cells labeled with PKH26 dye in the lymph nodes had rod-shaped BGs that had triple-layered limiting membrane in the Golgi area. Some LCs with atypical granules were observed in the regional lymph nodes (arrowhead) (Figure 3). Considering the results of the above-described PKC-II and PKH26 studies, it is apparent that the time point at which LCs bearing antigens started to migrate from the skin to the T-cell areas of lymph nodes was within 3 hours, and the replenishment period for the LCs might

Table 1. LCs number and GM-CSF.

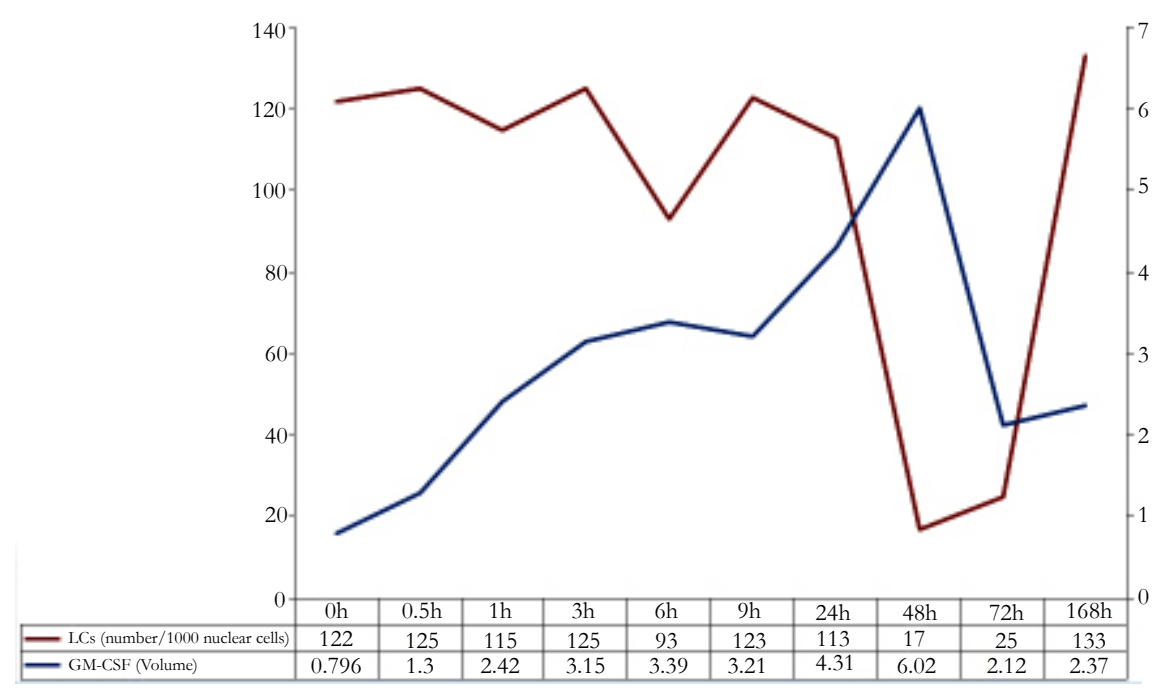


Figure 2.

A

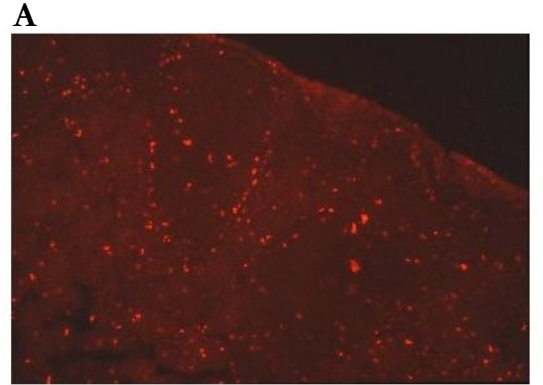

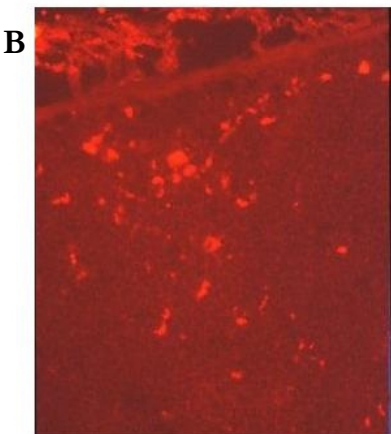

Figure 3. Atypical Granules (Arrow)

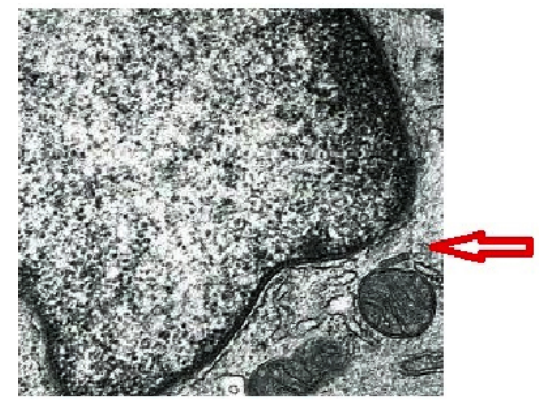

be from 72 to 168 hours.

In our study, LCs with atypical granules were observed in the lymph nodes at an early time point, most likely because the NC mice that were used have dry skin and are always stimulated by antigens; the LCs of these mice processed antigens quickly (the migration speed was about $1 \mathrm{~cm} / \mathrm{hr}$.) and they migrated with maturation.

Cytokines produced by $\mathrm{T}$ cells stimulate eosinophils, mast cells, and lymphocytes. Then these cells produce some cytokines and chemical mediators, and then degranulation from these cells amplifies allergic skin reactions. One essential factor in the viability and function of LCs is granulocyte-macrophage colony-stimulating factor (GM-CSF) [25]. Dermatophagoides farina (Def 1) may be related with allergic reaction by inducing production GM-CSF in keratinocytes [26]. When foreign substances contact to the skin, these substances stimulate keratinocytes. GM-CSF, which is produced by activated keratinocytes, causes the maturation and migration of LCs. Our studying showed that the volume of GM-CSF in the patch-testing sites increased with time and the peak was at a 48-hour site (Table 1). LCs migrate from the skin via lymph vessels [27], and these cells go to the lymph nodes with maturation. These migration and maturation are caused by GM-CSF.

\section{Conclusion}

Not only LCs migrate from the skin via lymph vessels but also the destination of LCs migration is to the lymph nodes for presenting antigens.

\section{References}

[1]. Murphy GF, Bhan AK, Sato S, Harrist TJ, Mihm MC (1981) Characterization of Langerhans cells by the use of monoclonal antibodies. Lab Invest 45(5): 465-468.

[2]. Hart DNJ (1997) Dendritic cells: Unique leukocyte populations which con- trol the primary immune response. Blood 90(9): 3245-3287.

[3]. Romani N, Schuler G (1992) The immunologic properties of epidermal langerhans cells as part of dendritic cell system. Springer Semin Immunopathol 13(3-4): 265-279.

[4]. Noordegraaf M, Flacher V, Stoitzner P, Clausen BE (2010) Functional Redundancy of Langerhans Cells and Langerin+ Dermal Dendritic Cells in Contact Hypersensitivity. J Invest Dermatol 130(12): 2752-2759.

[5]. Bennett CL, Noordegraaf M, Martina CA, Clausen BE (2007) Langerhans cells are required for efficient presentation of topically applied hapten to $\mathrm{T}$ cells. 179(10): 6830-6835.

[6]. Stingl G, Katz SI, Green I, Shevach EM (1980) The functional role of Langerhans cells. J Invest Dermatol 74(5): 315-318.

[7]. Kimber I, Cumberbatch M, Deraman RJ, Bhushan M, Griffiths CE (1999) Cytokines and chemokines in the initiation and regulation of epidermal langerhans cell mobilization. Br J Dermatol 142(3): 401-412.

[8]. Brand CU, Hunziker T, Limat A, Braathen LR (1993) Large increase of Langerhans cells in human skin lymph derived from irritant contact dermatitis. Br J Dermatol 128(2): 184-188.

[9]. Tuchinda P, Gaspari AA (2010) Langerhans cells in allergic contact dermatitis. G Ital Dermatol Venereol 145(6): 747-762.

[10]. Villablanca EJ, Mora JR (2008) A two-step model for Langerhans cell migration to skin-draining LN. Eur J Immunol 38(11): 2975-2980.

[11]. Randolph GJ, Beaulieu S, Pope M, Sugawara I, Hoffman L, et al (1998) A physiologic function for p-glycoprotein (MDR-1) during the migration of dendritic cells from skin via afferent lymphatic vessels. Proc Natl AcadSci USA 95(12): 6924-6929.

[12]. Villablanca EJ, Russo V, Mora JR (2008) Dendritic cell migration and lymphocyte homing imprinting. Histol Histopathol 23(7): 897-910.

[13]. Randolph GJ, Angeli V, Swartz MA (2005) Dendritic-cell trafficking to lymph nodes through lymphatic vessels. Nat Rev Immunol 5(8): 617-628.

[14]. Matsuda H, Kondo K (1997) A mouse model for atopic dermatitis. Clin Dermatol 51(Suppl 5): 41-45.

[15]. Ohara Y, Yonekawa H, Matsuda H (1997) A model mouse for atopic dermatitis. Molecular Medicine 34: 1554-1557.

[16]. Sugiura K, Hiramoto K, Shamoto M, Sugiura M, Hayakawa R, et al. (2004) Immunological cell situation in the skin of atopic model mice. J Eur Acad Dermatol Venereol 18(2): 148-152.

[17]. Sugiura K, Shamoto M, Sugiura M, Hayakawa R, Hata N, et al. (2000) The study on the role of Myocoptes musculinus (kekuidani) on the skin eruptions of the atopic model mice (NC mice). Environ Dermatol 7(2): 52-60.

[18]. Sugiura K, Sugiura M, Hayakawa R, Shamoto M, Takahashi H (2002) Study of the patch test reactions with the Myocoptes musculinus (Kekuidani) of NC/F mice (atopic model mice). Exog Dermatol 1(2): 87-91.

[19]. Magnusson B (1972) Patch testing. J Dermatol 82: 619-629.

[20]. Koyama Y, Hachiya T, Hagiwara M (1990) Expression of protein kinase C isozyme in epidermal Langerhans cells of the mouse. J Inv Dermatol 94(5): 677-680. 
[21]. Weinlich G, Heine M, Stössel H, Zanella M, Stoitzner P, et al. (1998) Entry into afferent lymphatics and maturation in situ of migrating murine cutaneous dendritic cells. J Inv Dermatol 110(4): 441-448.

[22]. Kobayashi M, Hashino T (1983) Distribution of cored tubule-containing Langerhans cells in the skin and Lymph nodes of mice. J Electron microscopy 32(3): 197-206.

[23]. Kripke ML, Munn CG, Jeevan A, Tang JM, Bucana C (1990) Evidence that cutaneous antigen-presenting cells migrate to regional lymph nodes sensitization. J Immunol 145(9): 2833-2838.

[24]. M Shamoto (1970) Langerhans cell granule in Letterer-Siwe disease. An electron microscopic study. Cancer 26(5): 1102-1108.

[25]. Witmer-Pack MD, Olivier W, Valinsky J, Schuler G, Steinman RM (1987)
Granulocyte / Macrophage Colony-Stimulating Factor is essential for the viability and function of cultured murine epidermal Langerhans cells. J Exp Med 166(5): 1484-1498.

[26]. Kimura T, Sekido M, Chimura N, Shibata S, Kondo N, et al. (2012) Production of GM-CSF mediated by cysteine protease of Der $\mathrm{f}$ in canine keratinocytes. J Vet Med Sci 74(8): 1033-1036.

[27]. Sugiura K, Shamoto M, Sakamoto N, Shinzato M, Osada A, et al. (2003) It is true that, when Langerhans Cells Migrate from the Skin to the Lymph Node, they are transported via Lymph Vessels. Dermatology 206(3): 222224. 